\title{
Simulating Urban Resilience: Disasters, Dynamics and (Synthetic) Data
}

\section{A. Yair Grinberger, Michal Lichter and Daniel Felsenstein}

\begin{abstract}
An agent based (AB) simulation model of urban dynamics following a disaster is presented. Data disaggregation is used to generate 'synthetic' data with accurate socio-economic profiling. Entire synthetic populations are extrapolated at the building scale from survey data. This data is coupled with the $\mathrm{AB}$ model. The disaggregated starting population allows for the bottomup formulation of the behavior of an entire urban system. Agent's interaction with each other and with the environment leads to change in residence and workplace, land use and house prices. The case of a hypothetical earthquake in the Jerusalem CBD is presented as an illustrative example. Dynamics are simulated for a period up to 3 years, post-disaster. Outcomes are measured in terms of global resilience measures, effects on residential and non-residential capital stock and population dynamics. The visualization of the complex outputs is illustrated using dynamic webmapping.
\end{abstract}

\footnotetext{
A.Y. Grinberger; M. Lichter; D. Felsenstein

The Hebrew University of Jerusalem, Jerusalem, Israel

e-mails: asherya.grinberger@mail.huji.ac.il

mlichter@geo.haifa.ac.il

daniel.felsenstein@mail.huji.ac.il
} 


\section{Introduction}

Urban resilience is invariably conceptualized as a cities' ability to 'bounce back', postdisaster, to some pre-existing equilibrium (Campanella, 2008; Godschalk, 2003; Müller, 2011). This pre-shock state embodies spatial and temporal relationships, direct and indirect effects and short and long term process. Disentangling these in order to isolate those factors promoting urban resilience is particularly challenging. In addition, urban resileince is more than just the sum of the parts of its' inhabitants' individual resilience. While cities are agglomerations of individuals, they are also much more. For example, they represent the accumulation of rounds of fixed capital expressed in infrastructure and other hard investment. These are generally expressed in terms of stock variables (roads, buildings etc). In the event of a shock, resilience of stock will be expressed in static terms, for example by maintaining function (transport flows, energy provision, providing shelter) in the face of adverse conditions.

However cities are also much more than accumulations of capital stock. They comprise complex network and flow systems such as input-output relations between producers and consumers, origin-destination traffic patterns and so on. These represent a dynamic and long term view of resilience that involves not just maintaining the existing state of the city but also recovering in order to reach a desired state. Flows of information, labour and capital have the ability to not just maintain current conditions but to change the urban growth trajectory by increasing productivity over the medium to long term . Dynamic resilience therefore contributes variable inputs to urban development and the more inputs are variable, the greater the likelihood of inefficient allocation of urban resources (Rose, 2009).

This chapter presents a disagregated agent-based (AB) simulation model of urban resilience in the wake of a disaster. The activities of multiple agents create a computable system in which the actions of individual agents affect each other and the system as a whole. The result is a complex network of behavior patterns that could not have been predicted by simply aggregating individual agent behavior. The system can be simulated and subjected to various exogenous shocks. The motivation for the study is to show how a bottom-up simulation modeling approach combined with an initial population created from synthetic data at the building scale, can be used to aid urban rejuvenation in the aftermath of a disaster. We also illustrate how web-based 
technology can be used for communicating these findings to planners, policy makers and the public.

\section{Agent-Based Modeling and Urban Disasters}

Urban disasters occur randomly in time and space. They affect both individuals and the environments that they populate. Large scale disasters are generally not one-time disturbances but generate a series of sub-incidents such as aftershocks in the case of earthquakes or secondary contamination in the case of pandemics. These keep the disaster environment in state of flux. Individuals therefore operate in a randomly changing context. By 'agentizing' this environment (Axtell, 2000) and reducing its elements to autonomous programmable entities, it becomes amenable for management.

Agent-based simulations have been applied in a variety of disaster contexts such as flooding, fires and earthquakes (Chen and Zhan, 2008; Crooks and Wise, 2013; Dawson et al., 2011). The AB framework lends itself to these situations. A high level of agent heterogeneity can be programmed and applied differentially to the various stages of an urban disaster from mitigation and preparation through response and on to recovery. This yields a rich array of human behaviors. For example, AB models have been coupled with network models for simulating evacuation (Chen, et al., 2012). GIS tools and crowdsourced data has been combined with agent based modeling to assist with post disaster recovery analysis (Crooks and Wise, 2013). Kwan and Lee (2005) merged network analysis, GIS and 3D visualization tools to provide a realtime micro-scale simulation tools for emergency response at the the individual building or city block level. In the field of traffic modeling, Chen and Zhan (2008) have used the AB approach to evaluate different evacuation strategies under different road network and population density regimes. The emergency response literature also uses $\mathrm{AB}$ modeling in urban contexts to provide a simulation capability for the public health and medical communities. This allows for the efficient management of medical and evacuation resources under conditions of severe uncertainty and stress .Invariably these systems use hybrid architecture that integrates a simulator with GIS, databases and rule based protocols for agents (Narzisi et al., 2006; Zimmerman et al 2010). 


\section{Method}

To account for the spatio-temporal dynamics of urban disasters we present an agent-based framework that is driven by synthetic spatial data (Figure 1). In this framework residential choice, workplace and activity location are determined bottom-up while land use and house prices are fixed top-down. As agents are inherently mobile, their behavioral response is articulated in varying temporal and spatial dimensions. While much of the data for observing agents behavior is only available at coarse administrative units, we use a GIS-based method for the accurate socioeconomic profiling of the population under such circumstances. This involves moving from a database describing only hundreds or thousands of spatial units to one containing records of millions of buildings and individuals over time. In the resultant spatial data, every individual in a city is synthetically represented by a single specific record. This database is input to an agentbased model of urban disasters as the initial physical properties of the urban environment and the distribution of the units (Figure 1). Repast Simphony 2.0, an open source, Java-based programming platform, is used as the simulator (Crooks and Castle, 2012). Model outputs are delivered and visualized using the tools of web-GIS. 
Fig. 1 Simulating Resilience: A General Framework

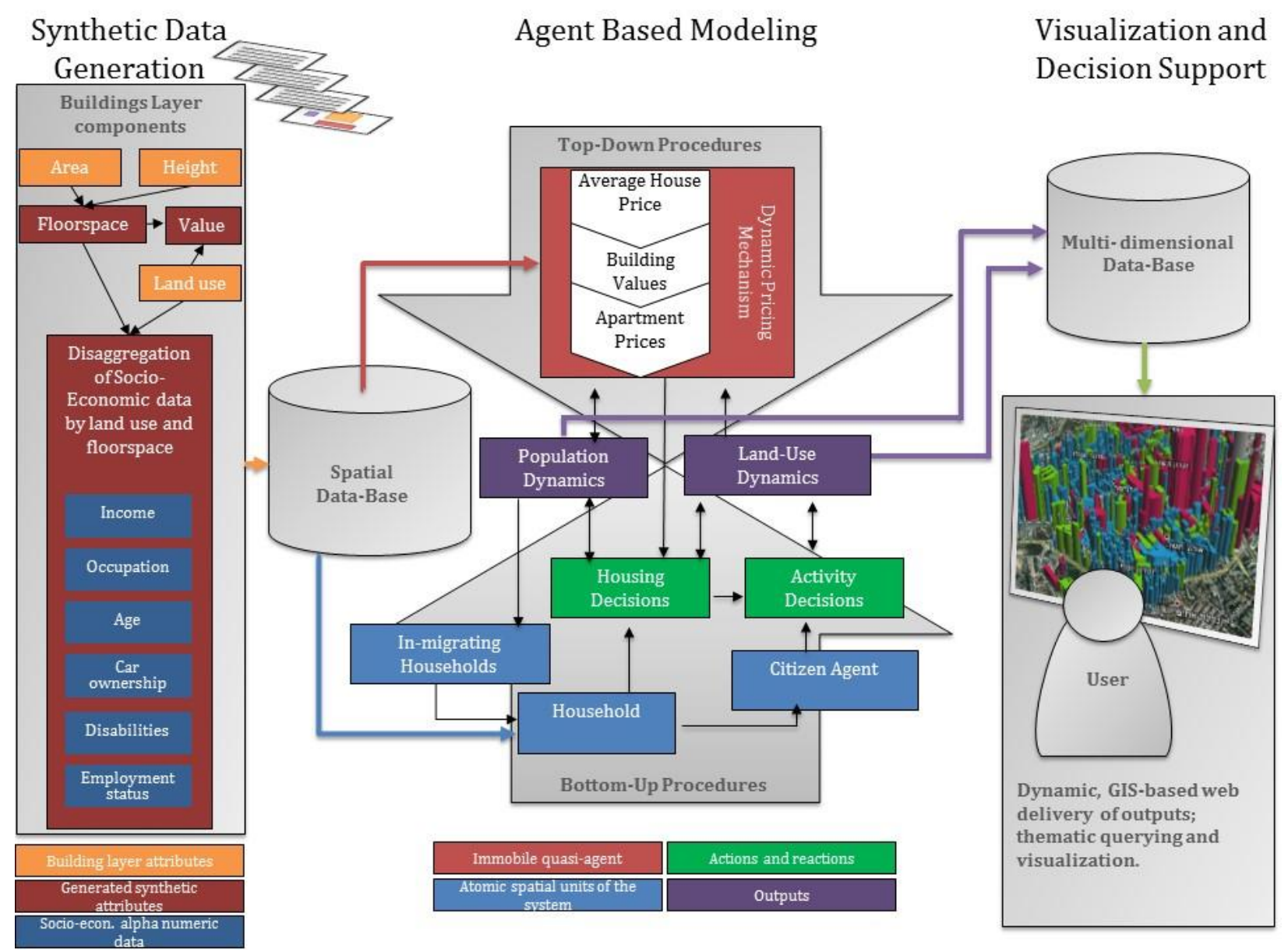

The urban system is modeled as the outcome of interactions between agents and the environment (Fig 1). In this section we describe the mechanics of the agent-based procedures that give the database a dynamic, multi-dimensional nature. These mechanics rely on (necessary) simplifying assumptions. While this limits realism, it is required for modeling an agent-rich environment. To increase the validity of results, the model's mechanics are 'structurally stochastic', as the random preference element allows behavior to vary in relation to the basic behavioral structure (Reichert and Mieleitner, 2009). While the assumptions we make are not ungrounded, the stochastic element diminishes possible diversion from realistic behavior, given the large number of agents in our model. Common validation processes (such as backstacking) are inapplicable in the case of long-term effects. Hence the need for a solid base for the mechanics of the model. 


\subsection{Behavior of Agents}

Citizen agents are organized at two levels - as individuals and clustered into households. Each agent embodies both socio-economic and spatial properties. Residential decisions are made at the household level while activities and workplace location decisions are executed by individuals. Agent behavior is not ad hoc but grounded in standard behavioral principles of utility maximization and risk evasiveness (Lancaster, 1966), satisficing (Simon, 1952), preferences for scale in economic activity (Fujita and Thisse, 2002) and segregative residential choice (Schelling, 1971). Residential and activity location decisions are guided by a search process grounded in 'satisficing' behavior. In this process the first location found to satisfy a set of constraints and a utility threshold that represents preferences, is chosen. This threshold is randomly drawn for each agent from the range $[0,1]$.

\subsubsection{Place of residence}

At any given moment, a household may decide to relocate or to move out of the city. In most cases this choice is probabilistic and dependent upon exogenous probabilities for outmigration/relocation. In exceptional cases a household is forced out of home due to land-use dynamics such as residential use becoming commercial or due to the direct destruction resulting from the disaster. In this case the choice between relocation and out-migration is entirely random. The choice of new place of residence (Eq 1) is guided by two elements: the affordability of a dwelling and its attractiveness (Chen et al., 2012). These are evaluated in relation to the household's willingness to allocate up to one third of monthly income to housing (a budget constraint) and preference for residential segregation represented by limited tolerance to change in living environment:

(1)

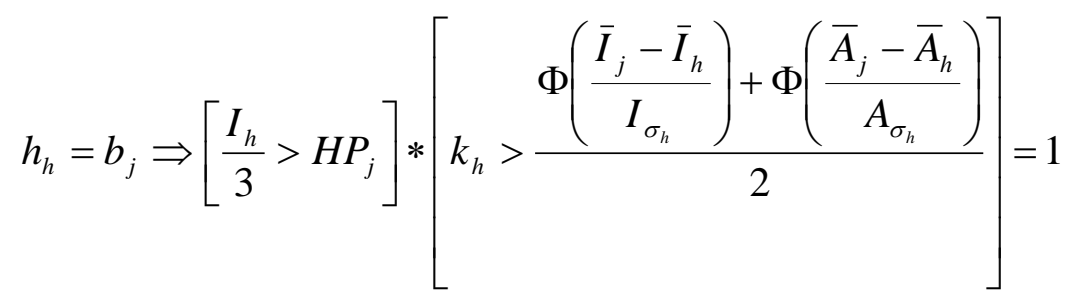

where:

$h_{h}$ is the new residential location for household $h$ randomly drawn from a choice set that includes all vacant buildings and partially occupied residential buildings, $b_{j}$ is the building considered, 
[ ] is a binary expression with value of 1 if true and 0 otherwise, $I_{h}$ is household $h$ 's monthly income,

$H P_{j}$ is monthly housing cost of an average housing unit in building $j$,

$k_{h}$ is the tolerance level for household $h$,

$\Phi$ is the standard normal cumulative probability function,

$\bar{I}_{j}, \bar{A}_{j}$ are the average household income and average age of individuals in building $j$, respectively

$\bar{I}_{h}, \bar{A}_{h}$ are average household income and average age of individuals in residential buildings within 100 meter of current residential location of household $h$,

$I_{\sigma_{h}}, A_{\sigma_{h}}$ are standard deviations of household income and of resident age in residential buildings within 100 meters from current home location of household $h$, respectively.

A random-order search process is initiated whenever a household relocates or when a new household migrates into the city. The volume of in-migration is proportional to the number of vacant dwelling spaces and to an exogenous ratio of in-migration to out-migration. The search process is terminated if 100 iterations do not lead to relocation. In this case the household (whether in-migrant or native) leaves the city. If the conditions for relocation are fulfilled, the dwelling unit is removed from the set of vacant units.

\subsubsection{Workplace location}

Location of workplace is related to land-use as each employment sector is associated with a particular use (e.g. commercial, industrial, governmental etc). Apart from this constraint, locational choice (Eq 2) is dependent on distance-minimizing and preference for scale (representing more opportunities):

(2)

$W P_{i}=b_{j} \Rightarrow\left[L U_{j}=E L U_{i}\right] *\left[k_{i}>\frac{D_{i j} / \max D_{i}+1-F S_{j} / \max F S}{2}\right]=1$

where:

$W P_{i}$ is the workplace location of individual $i$,

$E L U_{i}$ is the employment-sector-related land-use for individual $i$,

$k_{i}$ is the preferences index,

$D_{i j}$ is the distance between building $j$ and individual $i$ 's place of residence, 
$\max D_{i}$ is the distance of the building within the study area furthest away from individual $i$ 's place of residence.

Workplace is not part of the initial database and agents are assigned to locations within the model. These locations are assumed to be stable unless the building changes use. Only in such a case is the search for a new workplace initiated.

\subsubsection{Location of activities}

Each day an individual agent participates in a varying number of activities. This number is dependent on individual attributes promoting or inhibiting mobility and accessibility (e.g. age, car ownership, disability) as well as employment status and personal preferences:

$N A c_{i}=\| a *\left(\frac{k_{i}}{0.5}\right) *\left(1+\operatorname{car}_{h} * 0.33\right) *\left(1-\operatorname{dis}_{i} * 0.33\right) *\left(1+\left[\operatorname{age}_{i}=2\right] * 0.33\right) *\left(1-\left[\right.\right.$ age $\left.\left._{i} \neq 2\right] * 0.33\right) \|+$ emp $_{i} * \operatorname{loc}_{i}$ where:

$N A c_{i}$ is the number of activities for resident $i$, $k_{i}$ is a preference index, $\mathrm{car}_{h}$ is a binary variable equal to 1 if the household $h$ owns a car and 0 otherwise, $d i s_{i}$ is a binary variable equal to 1 if individual $i$ is disabled and 0 otherwise, $a g e_{i}$ is the age group of individual $i$, $e m p_{i}$ is a binary variable equal to 1 if $i$ is employed and 0 otherwise, $l o c_{i}$ is a binary variable equal to 1 when $i$ 's workplace is located within the study area and 0 otherwise, $\|x\|$ indicates the nearest integer number to $x$,

$a$ is the average number of activities based on employment status; equals 2.5 for employed residents and 3 for non-employed.

The number of activities thus ranges between 0 and 12. The location of each activity is set by distance from previous location (starting from home) minimizing preferences, risk evasive behavior and preferences for scale. Risk evasiveness is embodied in the tendency to avoid areas in which a large proportion of the buildings are vacant and volume of floor-space represents preferences for scale.

(4) $a_{t+1, i}=b_{j} \Rightarrow\left\lfloor b_{j} \neq a_{t, i}\right\rfloor *\left\lfloor k_{i} \geq \operatorname{Att}\left(b_{j}\right)\right]=1$

where:

$a_{t, i}$ is the current location of individual $i$,

$a_{t+1, i}$ is the next location of activity of individual $i$, 
$k_{i}$ is a randomly drawn number between $[0,1]$ reflecting activity location preferences, $A t t\left(b_{j}\right)$ is the attractiveness score for building $j$, calculated as follows:

$$
\operatorname{Att}\left(b_{j}\right)=\frac{1-\Sigma E_{j} / \Sigma B_{j}+1-D_{i j} / \max D_{i} *\left(1+0.33 *\left(-\operatorname{car}_{h}+\operatorname{dis}_{i}+\left[a g e_{i}=3\right]\right)\right)+\left[L U_{j}=n o n \operatorname{Re} s\right] * F S_{j} / \max F S}{2+\left[L U_{j}=\text { non } \operatorname{Re} s\right]}
$$

where:

$\Sigma E_{j}$ is the number of unoccupied buildings within a 100 meter buffer of building $j$,

$\Sigma B_{j}$ is the number of buildings within a 100 meter buffer of building $j$,

$D_{i j}$ is the distance of building $j$ from the current location of individual $i$,

$\max D_{i}$ is the distance of the building within the study area furthest away from the current location of individual $i$, $L U_{j}$ is the land-use of building $j$,

nonRes is non-residential use, $F S_{j}$ is the floor-space volume of building $j$,

$\max F S$ is the floor-space volume of the largest non-residential building within the study area.

\subsection{Environmental Processes}

In most agent-based models the environment is a passive backdrop which changes only as a direct consequence of agents' actions (for example, changes in resource levels due to consumption by agents). However many components of the urban environment while not proactive are at least reactive. For the sake of simplicity these are not modeled individually but in aggregate they are treated as components of environmental sensitivity. Individual spatial elements such as census tracts, buildings and dwelling units are characterized as quasi-agents. These are not autonomous or mobile but are sensitive to environmental changes. This mechanism operates topdown as the effects of aggregate trends trickle down to the level of individual quasi-agents. We employ this mechanism in the areas of land-use dynamics and housing prices.

\subsubsection{Commercial Land-Use Dynamics}

In the context of these dynamics, we enlist three assumptions. First, revenue levels required by a commercial function in order to be profitable are proportional to its floor-space volume. Second, actual revenues at a location are proportional to local flows of customers and third, flows 
are proportional to the traffic loads ${ }^{1}$ in the vicinity of the function. This set of assumptions allows for formalizing the (logistic) probability of land-use change (Eq 6) as related to the congruence between floor-space volume and traffic loads at a location (Eq 7). This congruence is formalized as the difference in the relative position within an exponential distribution (Eq 8), thus represents demand or supply surplus:

$$
\begin{aligned}
& \text { (6) } P_{j, t}\left(\Delta x_{j, t}\right)=\frac{e^{-\Delta x_{j, t}}}{1+e^{-\Delta x_{j, t}}} \\
& \Delta x_{j, t}=\frac{z_{T R_{j, t}}-z_{F S_{t, t}}}{\left|z_{F S}\right|} \\
& \text { (7) } \\
& \text { (8) } z_{y_{t, j}}=\hat{\lambda}_{t} *\left(e^{-\hat{\lambda}_{t} * y_{j, t}}-e^{-\hat{\lambda}_{t} * y_{\text {med }_{t}}}\right)
\end{aligned}
$$

where:

$P_{j, t}$ is the probability of land-use change for building $j$ at time $t$,

$\Delta x_{j, t}$ is the relative difference in standardized values for traffic load and floor-space for building $j$ at time $t$.

$z_{y_{j, t}}$ is the standardized value $y$ (in relation to the median value $y_{\text {med }}$ ) for building $j$ at time $t$ drawn from the exponential distribution $y \sim \operatorname{Exp}\left(\hat{\lambda}_{t}\right), \hat{\lambda}_{t}=\frac{1}{\bar{y}}$.

Traffic loads are calculated as the average daily load (citizens per meter) on roads within $100 \mathrm{~m}$ radius for the building at the preceding 30 days. $P$ values of 0.99 and above are set to represent a demand surplus. For such values residential or vacant buildings become commercial functions. In such a case, any residents in the location relocate according to principles discussed in section 3.1.1. Values within the range $[P(1)-0.01, P(1)]$ are used to identify supply surplus, or unprofitability, which results in commercial buildings becoming vacant. Such formalization and critical values limit the sensitivity of large commercial functions and small residential uses, thus eliminating a possible bias for changing initial uses in these cases.

\footnotetext{
${ }^{1}$ We do not employ a shortest-path algorithm for movement routes but use a computationally less-demanding model where agents move at each step to the not-already-visited node closest to the destination (in aerial distance; loops are removed from the path). This also represents satisficing behavior.
} 


\subsubsection{Dynamic House Pricing}

Housing decisions (Section 3.1.1) are made in relation to prices. These prices represent demand, supply and the locational (dis)advantages of a specific dwelling unit (DU) and building. In order to capture the unique contribution of such market-level dynamics to the value of the individual unit we formalize a three-stage mechanism. Within this mechanism the effects of global changes trickle down from the census-tract (CT) level to the building and DU level. Average housing values per meter in CTs (Eq 9) change daily with changes to supply, demand and accessibility to services (supply of non-residential functions) within them. These prices set the value of individual buildings, along with local accessibility levels (Eq 10). Assuming equal size for all DUs within a building, the monthly cost of housing is derived in relation to the average willingness to pay in the population ( $\mathrm{Eq} 11)$. Changes to commercial values are the result of a similar simpler process which is dependent on supply only and ends at the building level (see Eq 10 and $\mathrm{Eq} 11)$ :

(9) $A V_{C T, t+1}=A V_{C T, t} *\left(1+\log \left(\frac{\left(\operatorname{pop}_{C T, t+1} / \operatorname{pop}_{C T, t}+{ }^{r e s_{C T, t} / r e s_{C T, t+1}}\right) *[L U=\operatorname{Re} s]+n \operatorname{Re} s_{C T, t+1} / n \operatorname{Re} s_{C T, t}}{1+2 *[L U=\operatorname{Re} s]}\right)\right)$

where:

$A V_{C T, t}$ is average value (commercial or housing prices) per meter in $C T$ at time $t$, pop $_{C T, t}$ is population in $C T$ at time $t$, res $_{C T, t}$ is the number of residential buildings in $C T$ at time $t$, $n \operatorname{Re} s_{C T, t}$ is the number of non-residential buildings in $C T$ at time $t$, $[L U=\operatorname{Re} s]$ is a binary expression which is true (equals 1 ) if $A V$ relates to housing prices and 0 otherwise.

$$
V_{j, t}=A V_{C T, t} * F S_{j} *\left(S L_{j, t} / S L_{C T, t}\right)^{[L U=\operatorname{Re} s]}
$$

where:

$V_{j, t}$ is the house price of a dwelling unit in building $j$ at time $t$, 
$S L_{s, t}$ is the service level within area $s$ at time $t$ - the ratio of non-residential buildings to residential buildings in this perimeter.

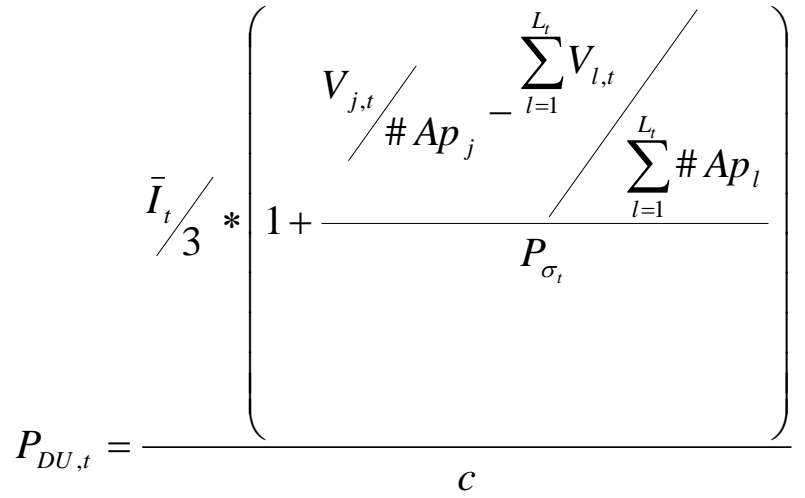

where:

$P_{D U, t}$ is the monthly cost of living in dwelling unit $D U$ at time $t$,

$\bar{I}_{t}$ is the average household income in the study area at time $t$,

\# Ap is the number of DUs within a building. If the building is initially residential, this is equal to its initial population size. Otherwise it is the floor-space volume of the building divided by 90 (the average DU size in meters),

$L_{t}$ is the number of residential buildings in the study area at time $t$,

$P_{\sigma_{t}}$ is the standard deviation of DU prices within the study area at time $t$,

$c$ is a constant.

\section{Context and Data}

The simulation involves a hypothetical earthquake in the CBD of Jerusalem. While the $\mathrm{CBD}$ lies in a relatively stable seismic area, the city itself is located only $30 \mathrm{~km}$ east of the active Dead Sea Fault Line. Moreover, the majority of the buildings in the city center were constructed prior to the institution of seismic-mitigation building regulations, hence they are prone to damage (Salamon et al., 2010). This study area covers $1.45 \mathrm{sq} \mathrm{km}$ and includes two major commercial spaces: the Mahaneh Yehuda enclosed street market and the CBD (see Figure 2). The heterogeneous mix of land uses is represented by residential buildings (243Th sqm, 717 structures and 22,243 inhabitants), commercial buildings (505Th sqm, 119 structures) and government/public use buildings (420Th sqm, 179 structures). Three major transportation arteries traverse the area and generate heavy traffic volumes: Agripas and Jaffa (light railway route) Streets run north-west to the south-east and King George Street runs north-south. 
The available data on buildings and population in the study area is aggregate data provided at the level of the Statistical Areas $(\mathrm{SA})^{2}$. The data drives the model at three different spatial resolutions: buildings, households and individuals. The variables used to populate the buildings can be grouped into three categories and are defined in Table 1:

- Building level: land-use, floor-space, number of floors, building value, households.

- Household level: inhabitants, earnings, car ownership.

- Individual level: Household membership, disability, participation in the work force, employment sector, age, workplace location.

Table 1 Variables used in the model

\begin{tabular}{|c|c|c|}
\hline Variable & Source & Spatial unit \\
\hline Residential building Value per $\mathrm{m}^{2}$ & National Tax authority 2008-2013 & SA \\
\hline $\begin{array}{l}\text { Non-residential plant and } \\
\text { machinery value }\end{array}$ & $\begin{array}{l}\text { Local authorities financial data } \\
\text { (CBS) (Beenstock et al., 2011) }\end{array}$ & Local authority \\
\hline Number of households & CBS 2008 & SA \\
\hline Number of inhabitants & CBS 2008 & SA \\
\hline $\begin{array}{l}\text { Average monthly household } \\
\text { earnings }\end{array}$ & $\begin{array}{l}\text { National Insurance Institute, annual } \\
\text { data }\end{array}$ & Local authority \\
\hline Labor force participation & CBS 2008 & SA \\
\hline Employment by sector & CBS 2008 & SA \\
\hline Percent disabled & CBS & SA \\
\hline Age & CBS & SA \\
\hline Workplace location & GPS survey 2014 & $\begin{array}{l}\text { survey of } \\
\text { individuals }\end{array}$ \\
\hline
\end{tabular}

We use a GIS buildings layer to provide the distribution of all buildings nationally with their inherit attributes such as aerial footprint, height and primary land use. These attributes are utilized to calculate the floor-space of each building. We then calculate the value of buildings

\footnotetext{
2 A statistical area (SA) is a uniform administrative spatial unit defined by the Israeli Central Bureau of Statistics (CBS). It corresponds to a census tract and has a relatively homogenous population of roughly 3,000 persons.
} 
according to their land use and floor space. Inhabitants socio-economic attributes are proportionally allocated to each building, using a methodology described in Lichter and Felsenstein (2012). The disaggregated building level data serves as the basis for the further disaggregation at the level of the individual. This begins with assigning each individual in the database a unique id, so that it is represented as a unique separate entity tied to a building in the database. Next, each person is allocated a random point location (a lat, lon coordinate) within the building with which it is associated. Demographic attributes (labor force participation, employment sector, disabilities and age group), assigned to buildings in the previous stage, are allocated to each individual so that they comprise the entire distribution in the building. Individuals are then clustered into households according to the household size (number of persons) in each building. Households are also represented as unique entities in the database and are associated with buildings. The clustering introduces heterogeneity in terms of the age distribution to closely represent a "traditional family household" having both adults and children when these are present in the building. A household entity represents the sum or average of the attributes of its members and is further assigned attributes such as earnings and car ownership in the same way these were assigned to individuals. The distribution of work locations of inhabitants by employment sector is derived from a GPS-based transport survey carried by the Jerusalem Transport Master Plan Team (Oliveira et al., 2011). We use this data to create a distribution of inhabitants working within and outside the study area, by sector of employment.

\subsection{Case Study Specifications}

The impact of the earthquake is modeled here as a one-time shock diffusing from a focal point. The effects of this shock result in physical damage to buildings and the road network. The probability that a building will collapse is proportional to its height and distance from the epicenter (Carenno et al., 2012) and is compared to a randomly-generated physical resilience score:

$$
b_{j}=\text { damaged } \Rightarrow\left[R_{j}<\frac{c * 10^{\text {mag }}}{D_{j} * \mid \log \left(D_{j}\right) * F_{j}}\right]=1
$$

where:

$R_{j}$ is the resilience score for building $j$,

$c$ is a constant, 
mag is the earthquake magnitude (similar to the Richter scale),

$D_{j}$ is distance of building $j$ from the earthquake epicenter,

$F_{j}$ is number of floors in building $j$.

Every building that collapses becomes vacant and unusable. All residents choose between migrating and relocating (see Section 3.1.1). A collapsed building blocks the closest road. Buildings are restored to pre-shock size and the duration required for this recovery (and the attendant re-opening of the blocked road) is proportional to floor-space (Carenno et al., 2012). Upon restoration, the building does not automatically retain pre-shock use.

The earthquake is simulated 25 times $^{3}$ with its epicenter randomly located in order to avoid location-based bias in the results. Each simulation comprises 1000 iterations (ticks) where each tick represented one activity day of resident-agents. In each simulation, the earthquake occurs after 50 iterations in order to let market dynamics kick-in and stabilize. The results below describe the averages of all simulations.

\section{Results}

The results presented below relate to both short and long term impacts. They differentiate between global impacts and their temporal and spatial distributions and between effects on population flows and housing stocks.

\subsection{Aggregate Patterns and Equilibrium}

Folke et al. (2002) conceptualize resilience as the ability of a system to reorganize itself following a change. This stresses the notion of moving beyond recovery to pre-shock state and attaining stability. The global indices of resilience presented below are constructed in this spirit. They quantitatively assess the tendency of a system to achieve a stable equilibrium. A system meets stability criteria if it registers consistent value levels over a consecutive period of days. Specifically, we relate to the difference between current value and the average value over the preceding 50 days. Attainment of equilibrium is defined as the earliest day (counting back from the end of the simulation) when the day-to-day change is not significant. Table 2 presents the

\footnotetext{
${ }^{3}$ This arbitrary number was chosen in order to balance between computing loads and convergence of results.
} 
frequencies of achieving equilibrium, along with average durations and final changes in value (final to pre-shock value ratio) for a variety of indicators.

Table 2 Global resilience measures

\begin{tabular}{|c|c|c|c|c|}
\hline Parameter & Variable & $\begin{array}{c}\text { Frequency of } \\
\text { Equilibrium (out } \\
\text { of } 25 \text { simulations) }\end{array}$ & $\begin{array}{c}\text { Average } \\
\text { Duration to } \\
\text { Achieve } \\
\text { Equilibrium } \\
\text { (days) } \\
\end{array}$ & $\begin{array}{c}\text { Average Final } \\
\text { Change }(\% \text { of } \\
\text { pre-shock } \\
\text { value })\end{array}$ \\
\hline \multirow{2}{*}{ Population } & Population & 24 & 397 & 67.85 \\
\hline & Average Income & 11 & 950 & 50.53 \\
\hline \multirow{2}{*}{$\begin{array}{l}\text { Residential } \\
\text { Stock }\end{array}$} & $\begin{array}{l}\text { Residential Stock Size (\# } \\
\text { buildings) }\end{array}$ & 25 & 332 & 88.34 \\
\hline & $\begin{array}{l}\text { Average Residential } \\
\text { Value }\end{array}$ & 22 & 677 & 96.12 \\
\hline \multirow{2}{*}{$\begin{array}{l}\text { Non- } \\
\text { Residential } \\
\text { Stock }\end{array}$} & $\begin{array}{l}\text { Non-Residential Stock } \\
\text { Size (\# buildings) }\end{array}$ & 23 & 670 & 142.43 \\
\hline & $\begin{array}{l}\text { Average Non-Residential } \\
\text { Value }\end{array}$ & 25 & 385 & 78.61 \\
\hline
\end{tabular}

The study area is resilient to the shock across most dimensions but not in the classical recovery sense of the term. Residential and non-residential capital stock both stabilize in terms of size (number of buildings) and value in the simulations but usually on values different to those existing under pre-shock conditions (the exception being average residential value). Population, the most mobile element in the model, does not show such stability. In the majority of cases (14/25, i.e. 56\%), income presents a continuing change, while in the other cases equilibrium is achieved quite late. This suggests that in spite of population size stabilizing, migration flows keep on affecting the composition of population.

The new situation of a larger, yet cheaper, non-residential capital stock is not fully explained by the decreasing value of non-residential stock, as stock size stabilizes long after values. Floor-space volume, which also affects values, may account for this trend as it decreases by $20 \%$ on average. Most of this decrease happens within 300 days of the shock and a negligible difference $(0.56 \%)$ is registered between values at day 350 and day 1000, correlating with the time required for non-residential values to achieve equilibrium. Therefore, these values experience a large initial shock. The new non-residential functions that appear over time are smaller in terms of floor-space thereby not further affecting values. 
Residential stock presents a mirror image with average values lagging behind size. Supply and demand (population size) dynamics do not temporally correlate with the recovery of average values. This suggests that these effects may be attributed to other elements in the housing price mechanism such as service levels (number of non-residential buildings). These stabilize just before residential values and floor-space volume. The latter increases by $12.7 \%$ on average. These two trends are sufficient to contain the effects of the sharp decrease in population thereby creating a recovery scenario in relation to housing values.

\subsection{Spatial and Temporal Distribution of Effects on Stocks}

As outlined in the previous section, the average post-shock picture is one of smaller and cheaper commercial functions along with slightly larger residential buildings. Yet inequalities in the distribution of these changes may exist, as some areas may enjoy/suffer their consequences more than others. Furthermore, these distributions may or may not be stable over time. Figure 2 represents the distribution of land-use over these two dimensions. The unequal spatial pattern is evident as the propensity for new commercial functions to emerge is greater in the areas southwest and north-east of the market. These new land uses tend to stabilize over time, as indicated by the diminishing vacancy rate. This phenomenon may be attributed to centripetal and centrifugal forces. The centrifugal force is set in motion by the physical damage of the earthquake blocking movement paths. In search of new routes, traffic patterns disperse from pre-shock state to the south-west and north-east ${ }^{4}$ (Figure 2, $\mathrm{T}=100$ ). As traffic loads are the locus behind the spatial pattern of commercial uses (see Section 3.2.1), their dispersal increases the potential profitability of new locations, attracting new activities away from previous clusters. Yet, the new emerging functions exert a self-enhancing centripetal force through a cyclical process of influence: new functions attract more traffic, which increases their profitability and attracts more uses, which further attract traffic loads. This centripetal force continues to work long after the effects of the initial shock have subsided thus perpetuating some of the new traffic patterns even after blocked roads start to open up ( $\mathrm{T}=250, \mathrm{~T}=1000)$.

\footnotetext{
${ }^{4}$ This is also apparent in change to the average standard deviation of traffic loads (agents per meter). Over time, the average s.d. decreases by $73.58 \%$ in relation to pre-shock state, suggesting a more even dispersal of traffic loads.
} 
Fig. 2 Spatial distribution of land-use change. Height represents the number of simulations in which landuse change (colored) and vacancy (in grey) occurs. Building color indicates initial land-use: Residential (green), Commercial (blue), or Public (pink). Road heights represent average traffic load. Roads in red denote values above the average

\section{$\mathrm{T}=50$}

Jaffa St.

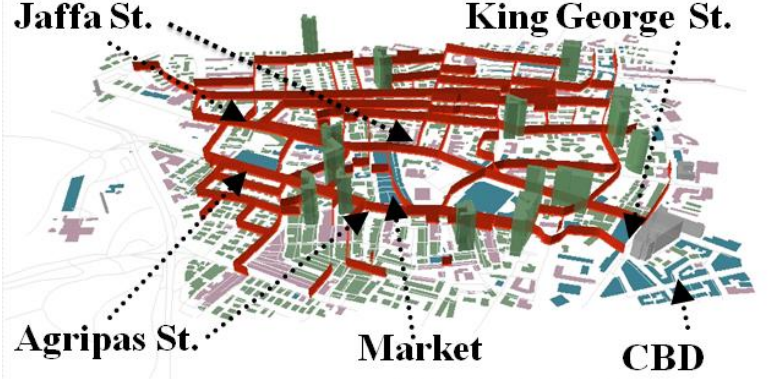

$\mathbf{T}=\mathbf{2 5 0}$

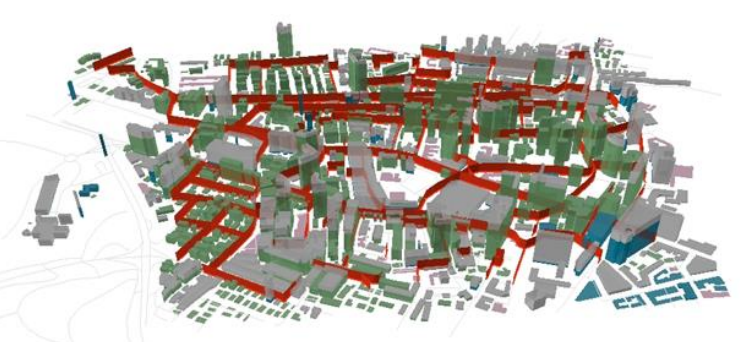

$T=100$

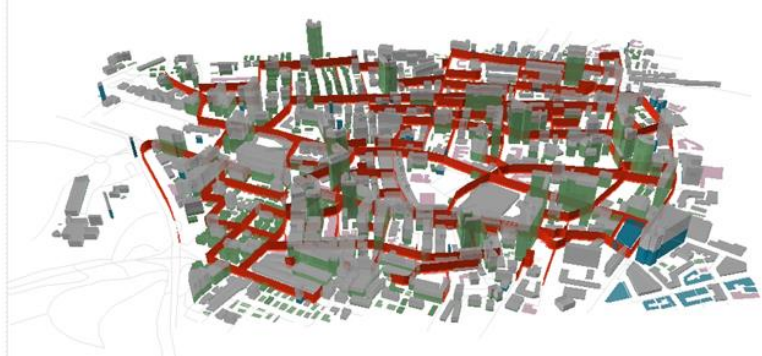

$\mathbf{T}=\mathbf{1 0 0 0}$

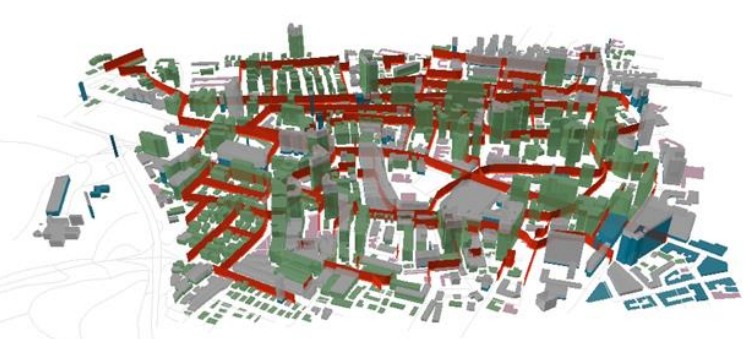

\subsection{Spatial and Temporal Population Dynamics}

In order to characterize population flows we calculate a normalized weighted composite 'Social Vulnerability Index' (SVI; see Lichter and Felsenstein, 2012) at the level of the building. The relative weights of the elements comprising the index reflect their contribution to aggregate socio-economic vulnerability.

$$
\begin{aligned}
& S V I_{b, t}=0.5 * \bar{I}_{b, t}+0.1 * C r_{b, t}-0.2 * \bar{A}_{b, t}-0.2 * D s_{b, t} \\
& \text { where: } \\
& \bar{I}_{b, t} \text { is the average monthly income of households residing in building } b \text { at time } t \text {, } \\
& C r_{b, t} \text { is the rate of car ownership of households residing in building } b \text { at time } t \text {, } \\
& \bar{A}_{b, t} \text { is the average age within households residing in building } b \text { at time } t \text {, } \\
& D s_{b, t} \text { is the share of residents who suffer disability building } b \text { at time } t .
\end{aligned}
$$


Assessing the spatio-temporal distribution of population flows is achieved via a two-step procedure. First, we interpolate the individual SVI values of buildings onto a continuous surface ${ }^{5}$ and then we calculate the Local Indicators of Spatial Autocorrelation ${ }^{6}$ (LISA; Anselin, 1995) for each cell. We do this at temporal intervals of 50 days. This procedure allows for identifying clustering and dispersal patterns over time. Figure 3 shows the significant clusters of similar values (high LISA values).

Fig. 3 Spatio-temporal distribution of SVI. Purple indicates higher absolute SVI values, brown indicates lower. Height represents LISA values. Building color indicates land-use at time $\mathrm{T}$ in the majority of the simulations: Residential (green), Commercial (blue), or Public (pink)
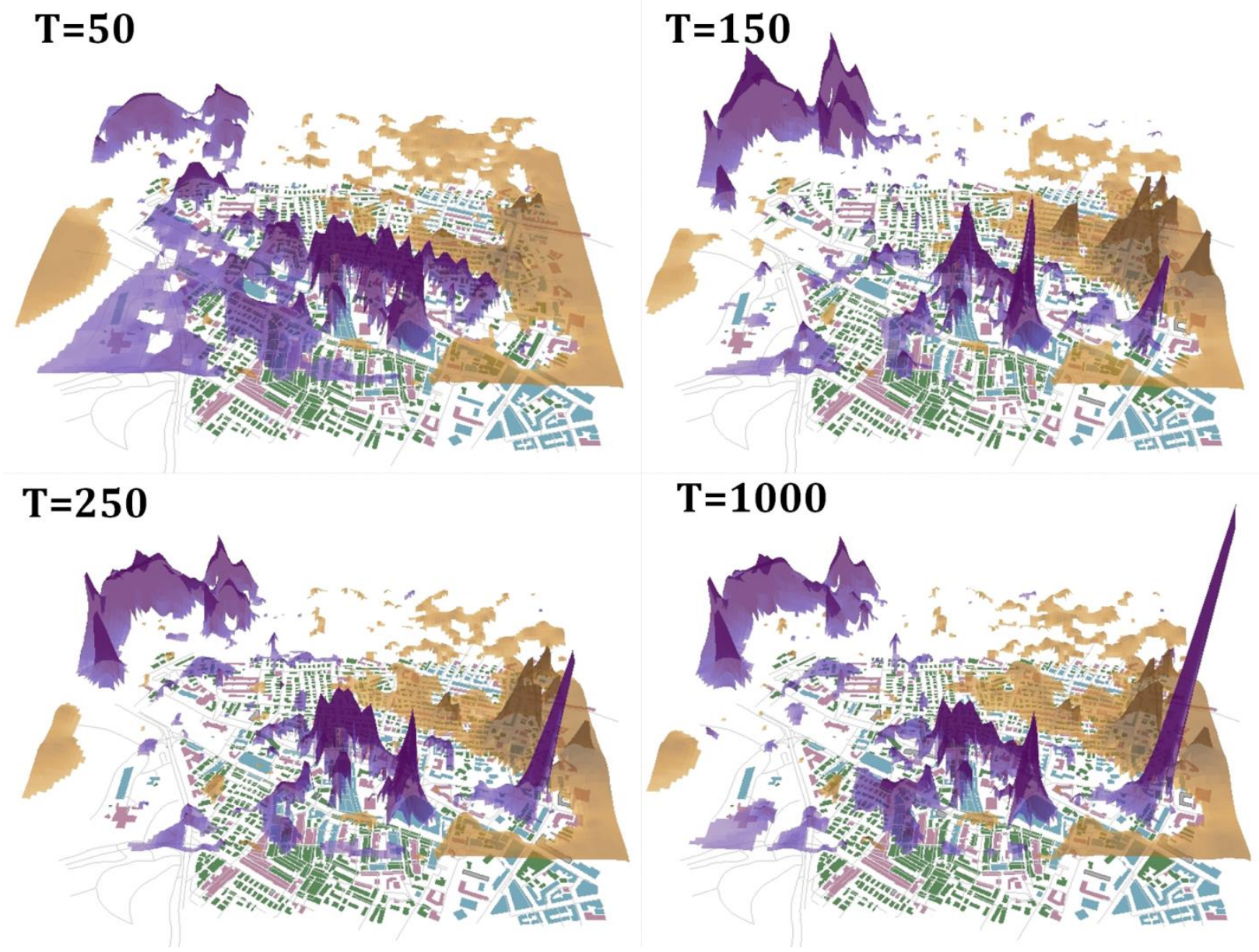

\footnotetext{
${ }^{5}$ We use an Inverse Distance Weighting procedure. The parameters used are: pixels of 10X10m, 100m search radius and $2^{\text {nd }}$ order power function.

${ }^{6}$ Neighborhood is defined using a queen contingency matrix.
} 
As in the case of land use, the results suggest the existence of a similar centripetalcentrifugal tension for population flows. The shock breaks down the initial divide of a less vulnerable western cluster and a vulnerable eastern areas $(\mathrm{T}=50)$ into a pattern of clusters surrounded by areas of mixed population $(T=150)$. The tendency of households to choose living environments that preserve previous conditions (see Section 3.1.1) acts as a centripetal force that makes pre-shock clusters more attractive. As the centrifugal effect of the shock is only temporary, clustering continues and the clusters grow and become more punctuated ( $\mathrm{T}=250$ and $\mathrm{T}=1000$ ).

In spite of these similarities, there is a subtle yet important difference between effects on flows and on stocks. While the dissolution of previous clusters into new agglomerations may be interpreted as a sign of recovery and efficiency in an economic system, in the social context this is not necessarily the case. This process of re-grouping may in fact exacerbate vulnerability as existing communal support systems may stop functioning. This is especially true in the case of vulnerable populations. As they are less mobile due to greater budget constraints, they are exposed to the effects of more resilient, in-migrant households. If such households are characterized by high tolerance to change, they can easily relocate to more vulnerable areas. By doing so they act as agglomeration nuclei, changing the nature of their environment and attracting more population similar to them. As social mixing increases, social cohesiveness of the neighborhood decays and its institutions break down. Vulnerable populations are thus faced with the choice of remaining with no support or migrating to 'ghettos'.

\subsection{Interactive Web-GIS application for visualization of results}

As the simulation outputs are multi-dimensional and include vast amounts of information on urban dynamics both spatial and temporal, we use web-GIS to communicate the results. The complexity of outputs is hard to internalize or visualize in their entirety using traditional graphic representations. We develop a web-based application to allow interactive visualization and querying of the multi-dimensional output in an intuitive and user-friendly fashion. (see http://ccg.huji.ac.il/AgentBasedUrbanDisaster/index.html). The site serves solely as a visualization tool for pre generated results and not as a vehicle for distributing the model. Using a simple web browser, users can generate time lapse visualizations in the form of maps and charts without any previous knowledge in handling spatial data or using GIS. They can choose a variable of interest, visualize its change over space and time and create location-specific information. This 
interaction is facilitated by simply clicking on the map or the chart or using gauges such as buttons and sliders (Fig 4). When initiating such an event, a single click can trigger complex querying of the database in the background. This necessitates database design and construction in a way that allows for fast and efficient data extraction. We create a dedicated database for the output results of time series from the model simulation. This is achieved by using DB design that does not always follow strict design guidelines but rather contains some flat tables to enable lateral data charting, displayed in pop-ups, graphs and charts. The visualization includes time lapse representation of human mobility (household level), changes in passengers along roads, changes in building land use and value, household socio-economic change and so on.

Fig. 4 Web-based querying and visualization application of selected variables on a dynamic webmap (see http://ccg.huji.ac.il/AgentBasedUrbanDisaster/index.html)

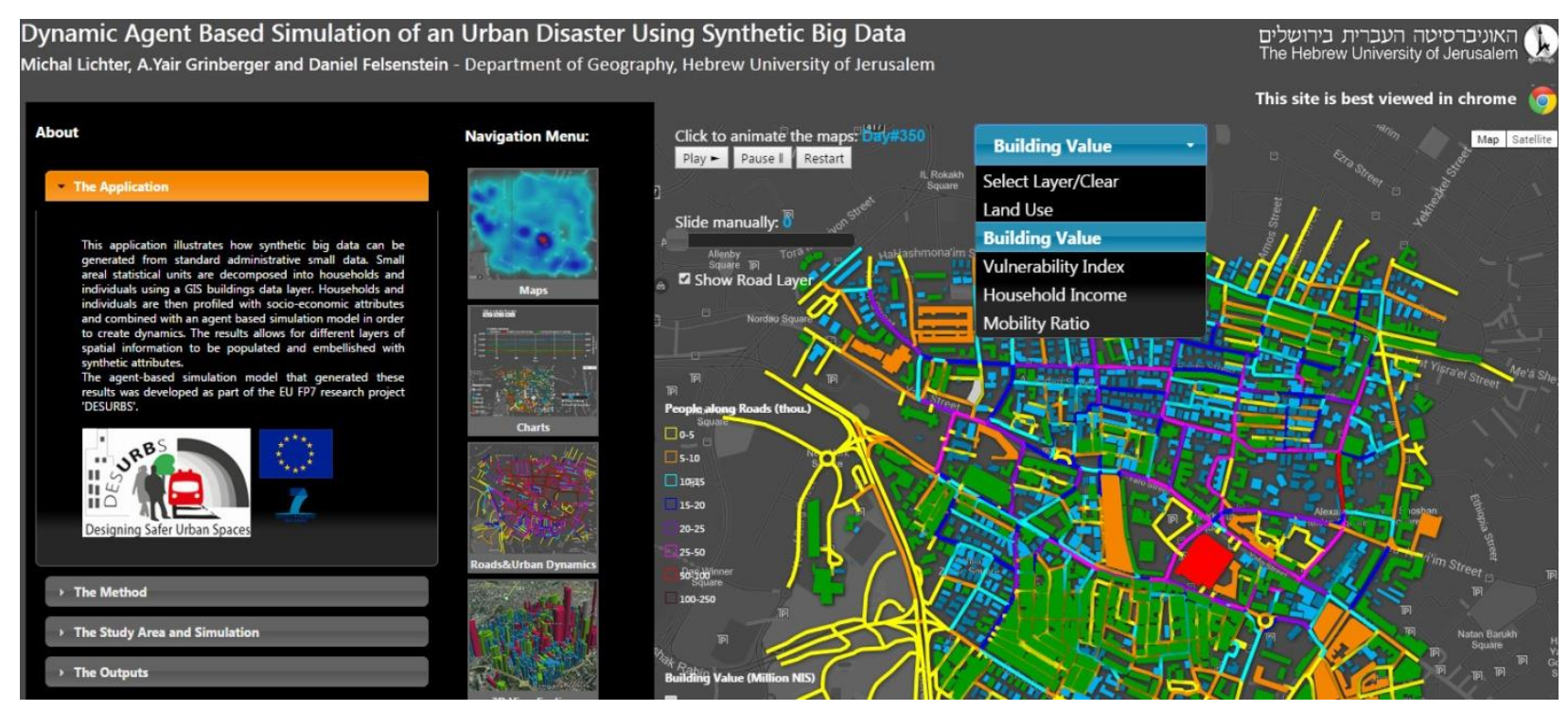

The web-mapping platform is Google Maps API. Middleware functionalities are added to the application based on JavaScript libraries and APIs. These functions interact with the webmapping platform to provide ancillary capabilities (Batty et al., 2010) such as time laps animation, action buttons, sliders, interactive graphs etc.

\section{Conclusions}

The findings above have looked at urban resilience at both the metropolitan and local scales. With respect to the former, we observe that the resilience patterns of residential and non- 
residential (commercial) capital stock are very different. Post-disaster, non-residential stock attains equilibrium based on a pattern of smaller, less expensive units, while the opposite holds for residential stock. Population levels stabilize much faster than incomes indicating demographic turnover and churning. At the local scale we look at the difference between the resilience of stock variables, exemplified by land use and that of flow variables, represented by a composite measure of social vulnerability. Our main finding is that in the advent of a shock, both stock and flow variables disperse and re-aggregate over time. However more resilient socio-economic groups cope better with dispersing and then re-clustering. Less resilient populations are more in need of community support systems and cannot rejuvenate quickly.

To add further realism, future work will need to relax some of the strong behavioral assumptions underlying the model. For example, the demand for housing is currently determined by affordability and attractiveness of the units on offer. This is a slightly mechanistic representation of a process that generally involves bidding, expectations and perceptions of opportunities. On the supply side, it would be useful to explicitly include the behavior of building contractors. At present, housing supply is driven by land use change and in particular by commercial land use becoming residential. Furthermore, migration behavior in the model is currently motivated by steady-state probabilities of movement augmented by the destruction of buildings. This results in mass flight followed by stabilization at a lower level equilibrium. We do not capture the psychological over-reaction of population movement identified in the literature (Stein et al 2010, Whitehead et al 2000). In this respect, our results may be downwardly-biased.

If stronger populations have the resources to accommodate the negative impacts of a disaster, then urban resilience is thus as much about economic welfare as it is about engineering or morphology. From a socioeconomic perspective, it is not the magnitude of the disaster that is important but the ability to cope with its results. Vulnerable populations or communities can be disproportionately affected by unanticipated disasters which are more likely to push them into crisis relative to the general population. Much of this can only be detected at the micro level such as the household or building. This is often smoke-screened in studies dealing with aggregate citywide impacts. The use of highly disaggregated and accurately profiled data is thus critical in understanding urban resilience. 
Two policy implications arise from the above findings. First, as an exogenous shock has no predetermined outcomes, a disaster may elicit wildly diverging responses in different urban environments. This has policy implications calling into question much of the popular literature advocating a 'one size fits all' approach to urban resilience (Prasad et al., 2009; UNISDR, 2012). While well intentioned, the standard check-list approach to promoting resilience may be misleading. Second, the dynamic simulation outcomes point to differential rates of recovery over time across the components of the urban system (for example residential and commercial capital stock). In an effort to 'get things done' in the aftermath of a disaster, public policy for urban recovery is often characterized by knee-jerk (over) reaction that involves time-compressing rebuilding and rejuvenation measures (Olshansky et al., 2012). The redevelopment opportunities for large scale urban change over a short period of time afforded by disaster, fail to recognize the existence of multiple and unstable urban equilibria resulting from different activities recovering at different rates. Urban resilience is as much about judiciously synchronizing recovery across the urban system as it is about getting cities to 'bounce back'.

Acknowledgements. Thanks to two referees for valuable comments on an early draft.

\section{Bibliography}


Anselin, L. (1995). Local indicators of spatial association - LISA. Geographical Analysis, 27(2). 93-115.

Axtell, R. (2000). Why Agents? On the varied Motivations for Agent Computing in the Social Sciences (Working Paper 17). Washington, DC: Center on Social and Economic Dynamics. Retrieved March 1, 2015, from http://www2.econ.iastate.edu/tesfatsi/WhyAgents.RAxtell2000.pdf.

Batty, M., Hudson-Smith, A., Milton, R., \& Crooks, A. (2010). Map mashups, web 2.0 and the GIS revolution. Annals of GIS, 16(1), 1-13.

Beenstock, M., Felsenstein, D., \& Ben Zeev, N. (2011). Capital deepening and regional inequality: an empirical analysis. Annals of Regional Science, 47(3), 599-617.

Campanella, T.J. (2008). Urban resilience and the recovery of New Orleans. Journal of the American Planning Association, 72(2), 141-146.

Carenno, M.L., Cardona, O.D., \& Barbat, A.H. (2012). New methodology for urban seismic risk assessment from a holistic perspective. Bulletin of Earthquake Engineering, 10, 547 -565.

Chen, X., \& Zhan, F.B. (2008). Agent-based modeling and simulation of urban evacuation: Relative effectiveness of simultaneous and staged evacuation strategies. Journal of the Operational Research Society, 59(1), 25-33.

Chen, Y., Li, X., Wang, S., \& Liu, X. (2012). Defining agents' behavior based on urban economic theory to simulate complex urban residential dynamics. International Journal of Geographic Information Systems, 26(7), 1155-1172.

Crooks, A.T., \& Castle, C.J.E. (2012). The Integration of agent-based modeling and geographical information for geospatial simulation. In A.J. Heppenstall, A.T. Crooks, L.M. See, \& M. Batty (Eds.), Agent-based models of geographical systems (pp. 219-251). Dordrecht: Springer.

Crooks, A.T., \& Wise, S. (2013). GIS and agent based models for humanitarian assistance. Computers Environment and Urban Systems, 41, 100-111.

Dawson, R,J., Peppe, R., \& Wang, M. (2011). An agent-based model for risk-based flood incident management. Natural Hazards, 59(1), 167-189.

Folke, C., Carpenter, S., Elmqvist, T,, Gunderson, L., Holling. C.S., \& Walker. B. (2002). Resilience and sustainable development: building adaptive capacity in a world of transformations. AMBIO: A Journal of the Human Environment, 31(5), 437-440.

Fujita, M., \& Thisse, J.F. (2002). Economics of Agglomeration: Cities, Industrial Location and Regional Growth. Cambridge, UK: Cambridge University Press.

Godschalk, D.R. (2003). Urban hazard mitigation: creating resilient cities. Natural Hazards Review, 4(3), 136-143. 
Kwan, M.P., \& Lee, J. (2005). Emergency response after 9/11: the potential of real-time 3D GIS for quick emergency response in micro-spatial environments. Computers, Environment and Urban Systems, 29(2), 93-113.

Lancaster, K.J. (1996). A New Approach to Consumer Theory. Journal of Political economy, 74, 132-157.

Lichter, M., \& Felsenstein, D. (2012). Assessing the cost of sea-level rise and extreme flooding at the local level: A GIS-based approach. Ocean \& Coastal Management, 59, 47-62.

Müller, B. (2011). Urban and regional resilience - A new catchword or a consistent concept for research and practice? In B. Müller (Ed.) German annual of spatial research and policy 2010 (pp. 1-13). Berlin-Heidelberg: Springer.

Narzisi, G., Mysore, V., Rekow, D., Triola, M., Halcomb, L., Portelli, I., \& Mishra, B. (2006 ). Complexities, catastrophes and cities: unraveling emergency dynamics. In: H. Schärfe, P. Hitzler, \& P. Øhrstrøm (Eds.) International conference on complex systems, Boston, MA, June 2006. Lecture notes in computer science (Lecture notes in artificial intelligence), vol 4068. Heidelberg: Springer.

Oliveira, M.G.S., Vovsha, P., Wolf, J., Birotker, Y., Givon, D. \& Paasche, J. (2011). Global positioning system-assisted prompted recall household travel survey to support development of advanced travel model in Jerusalem, Israel. Transportation Research Record: Journal of the Transportation Research Board, 2246(1), 16-23.

Olshanky, R.B., Hopkins, L.D., \& Johnson, L. (2012). Disaster and recovery: processes compressed in time. Natural Hazards Review, 13(3), 173-178.

Prasad, N., Ranghieri, F., Shah, F., Trohanis, Z., Kessler, E., \& Sinha, R. (2009). Climate resilient cities: A primer on reducing vulnerabilities to disasters. Washington, DC: World Bank publications.

Reichert, P., \& Mieleitner, J. (2009). Analyzing input and structural uncertainty of nonlinear dynamic models with stochastic, time-dependent parameters. Water Resources Research, 45(10), W10402. doi:10.1029/2009WR007814.

Rose, A. (2009). Economic resilience to disasters (Community and Regional Resilience Research Report 8). Oak Ridge, TN: Oak Ridge National Laboratory. Retrieved March 1, 2015, from http://research.create.usc.edu/published_papers/75.

Salamon, A., Katz, O., \& Crouvi, O. (2010). Zones of required investigation for earthquake-related hazards in Jerusalem. Natural Hazards, 53(2), 375-406.

Schelling, T. (1971). Dynamic Models of Segregation. Journal of Mathematical Sociology, 1(2), 143-186.

Simon, H. (1952). A behavioral model of rational choice. Quarterly Journal of Economics, 69(1), 99-118. 
Stein, R.M., Duenas-Osorio, L., \& Subramanian, D. (2010). Who Evacuates When Hurricanes Approach? The Role of Risk, Information and Location. Social Science Quarterly, 91(3), 816-834.

UNISDR (United Nations International Strategy for Disaster Reduction) (2012). How to make cities more resilient - a handbook for local government leaders. Geneva, Switzerland: UNISDR.

Whitehead, J.C., Edwards, B., Van Willigen, M., Maiolo, J., Wilson, K., \& Smith, K.T. (2000). Heading for higher ground: Factors affecting real and hypothetical hurricane evacuation behavior. Environmental Hazards, 2, 133-142.

Zimmerman, B., Nawn, D., Wang, Y., Kuhlman, B., Sochats, K., Luangesorn, L., et al. (2010). Dynamic Model Generation for Agent-Based Emergency Response Simulation. ESRI International User Conference, Center for National Preparedness, University of Pittsburgh. 